\title{
PHOTOLUMINESCENCE STUDIES \\ OF CUBIC PHASE GaN \\ GROWN BY MOLECULAR BEAM EPITAXY \\ ON (001) SILICON COVERED WITH SiC LAYER
}

\author{
M. Godlewski, V.Yu. IVANov \\ Institute of Physics, Polish Academy of Sciences \\ Al. Lotników 32/46, 02-668 Warsaw, Poland \\ J.P. Bergman, B. Monemar
}

Dept. Phys. and Meas. Technology, Linköping University, 58183 Linköping, Sweden

\author{
A. BARSKI AND R. LANGER
}

CEA/Grenoble, DRFMC/SP2M, 17 r. des Martyrs, 38054 Grenoble, France

In this work we evaluate optical properties of cubic phase GaN epilayers grown on top of (001) silicon substrate prepared by a new process. Prior to the growth $\mathrm{Si}$ substrate was annealed at $1300-1400^{\circ} \mathrm{C}$ in propane. The so-prepared substrate is covered with a thin $(\approx 4 \mathrm{~nm}) \mathrm{SiC}$ wafer, which allowed a successful growth of good morphological quality cubic phase GaN epilayers. The present results confirm recent suggestion on smaller ionization energies of acceptors in cubic phase $\mathrm{GaN}$ epilayers.

PACS numbers: 71.55.Eq, 78.47.+p

\section{Introduction}

Cubic phase $\mathrm{GaN}$ (c-GaN) epilayers show several interesting properties, which may lead to new applications of GaN-based devices. Two of these properties seem to be especially important. By the first we mean here the recent prediction on a higher optical gain for the c-GaN [1]. The second property was reported very recently by Menninger et al. [2]. These authors claim that the ionization energy of shallow acceptors in c-GaN is of about $100 \mathrm{meV}$, i.e., it is considerably smaller than for acceptors in wurtzite phase $\mathrm{GaN}$ ( $\mathrm{w}-\mathrm{GaN})$. Smaller acceptors ionization energy may result in a higher free hole concentration at room temperature.

\section{Samples}

Metastable c-GaN epilayers were grown on $\mathrm{Si}$ [3], $\mathrm{SiC}$ [4], $\mathrm{MgO}$ [5] and $\mathrm{GaAs}$ [6] substrates. In this work we describe optical properties of c-GaN epilayers grown by the molecular beam epitaxy (ECR-MBE) on top of (001) Si substrate, 
which prior to the growth process was annealed at $1300-1400^{\circ} \mathrm{C}$ in propane. This annealing covered $\mathrm{Si}$ substrate with a thin $(\approx 4 \mathrm{~nm}$ thick) SiC wafer acting as a buffer layer. Even though $\mathrm{SiC}$ layer is very thin, the so-obtained c-GaN epilayers are of a good morphological quality [7].

\section{Experimental results}

In Fig. 1 we show the photoluminescence (PL) spectrum of the GaN/SiC/Si epilayer measured at $12 \mathrm{~K}$ under uv line excitation of $\mathrm{Ar}^{+}$laser. In addition to the band-edge emission a weak "orange" PL was observed (inset), which is an analog of a known "yellow" PL of w-GaN samples, shown for the comparison. The band-edge part of the PL can be decomposed to four PL subbands with the maxima at $3.251 \mathrm{eV}, 3.191 \mathrm{eV}, 3.116 \mathrm{eV}$ and $3.034 \mathrm{eV}$. Two latter PL bands are noticeably broader than the first two.

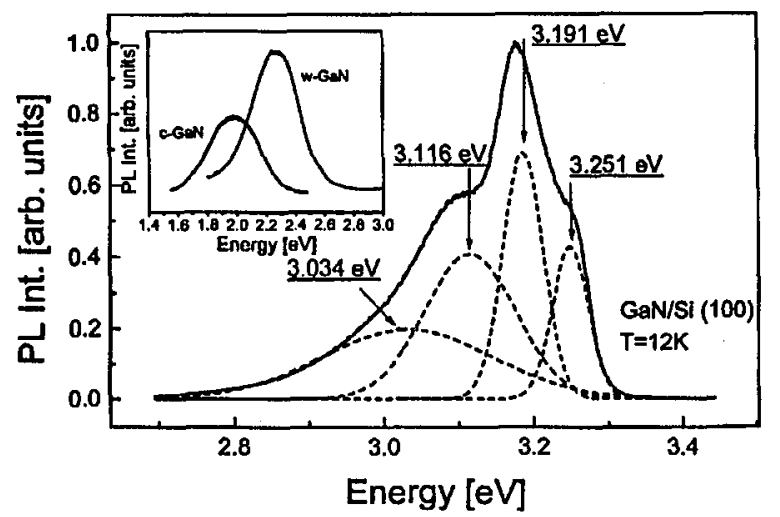

Fig. 1. Photoluminescence spectrum of the cubic phase $\mathrm{GaN} / \mathrm{SiC} / \mathrm{Si}(100)$ epilayer measured at $12 \mathrm{~K}$. In the inset we show a weak orange emission.

Intensity and temperature dependencies of PL and PL kinetics were measured to identify the PL subbands. PL bands, with the maxima at $3.034 \mathrm{eV}$ and $3.191 \mathrm{eV}$, increase linearly with the increasing excitation intensity. Two other PLs saturate at the increased excitation intensity. Spectral position of the $3.251 \mathrm{eV}$ and $3.191 \mathrm{eV}$ PLs does not change with an increase in the excitation intensity. For the $3.116 \mathrm{eV}$ PL a weak up in the energy shift was observed, which is more pronounced for the $3.034 \mathrm{eV}$ PL. Such up in the energy shift of the PL spectral position is expected for donor-acceptor pair (DAP) transitions, since for distant DAPs recombination rate is small and their contribution to the PL can be saturated at the increased light intensity.

The $3.191 \mathrm{eV} \mathrm{PL}$ and $3.034 \mathrm{eV}$ PL increase in the intensity up to $20-30 \mathrm{~K}$. For higher temperatures all PL subbands decrease in the intensity. For the temperature above $50 \mathrm{~K}$ this decrease is a single exponential and is characterized by the deactivation energies of about $30 \mathrm{meV}$ for the $3.191 \mathrm{eV} \mathrm{PL}, 20 \mathrm{meV}$ for the $3.116 \mathrm{eV}$ and $3.034 \mathrm{eV}$ PLs and of about $12 \mathrm{meV}$ for the $3.251 \mathrm{eV}$ PL. The decrease in the PL intensity is accompanied by a small shift of the spectral position of the 


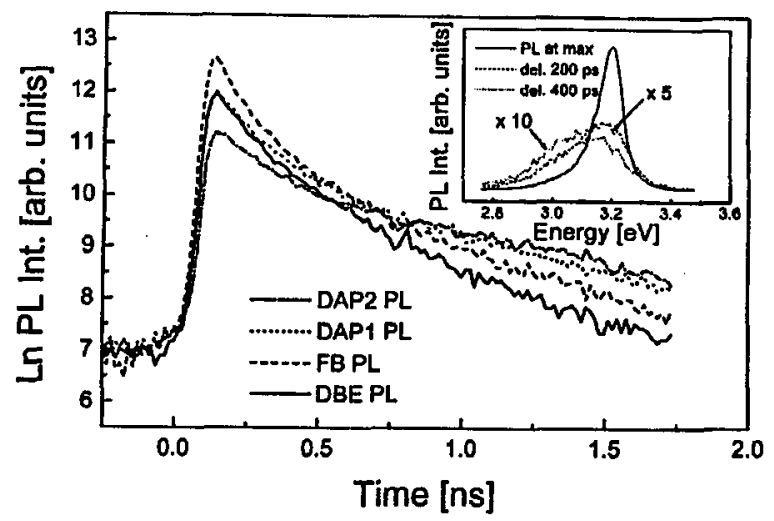

Fig. 2. PL kinetics and time-resolved PL spectrum (inset) of the cubic phase GaN/ $\mathrm{SiC} / \mathrm{Si}(100)$ epilayer measured at $2 \mathrm{~K}$ under nonresonant excitation conditions.

PL emissions. Whereas three other PLs shift down in the energy, which reflects the decrease in the band gap energy, the $3.191 \mathrm{eV}$ PL shifts up in the energy at increased temperature.

Picosecond dynamics of the edge-band PLs of c-GaN (GaN/GaAs) was first studied by Klann et al. in 1995 [8]. A biexponential decay of the free exciton (FE) PL was observed with an initial fast decay of about $15-40 \mathrm{ps,} \mathrm{which} \mathrm{was} \mathrm{related} \mathrm{to}$ FE relaxation to donor bound exciton (DBE). For DBEs a longer PL decay time was observed of about 100-400 ps. In Fig. 2 we show PL kinetics measured for the $\mathrm{GaN} / \mathrm{SiC} / \mathrm{Si}$ structure studied. Figure 2 indicates quite different PL decay times for four PL subbands. A biexponential PL decay is observed for all four emissions. All of them show a fast component of the decay $(\approx 200$ ps for the $3.251 \mathrm{eV}$ PL and $\approx 125 \mathrm{ps}$ for the $3.191 \mathrm{eV} \mathrm{PL}$ ) and a slow component (600-950 ps depending on the detection energy) observed at longer delay times. We relate the slow component of the decay of two higher energy PLs to their spectral overlap with the two low energy PL subbands showing longer PL decay times.

Due to different PL decay times, the PL changes its spectral shape at longer delay times in the time-resolved experiment shown in the inset of Fig. 2. At longer delay times, after the $3 \mathrm{ps}$ long laser pulse, the contribution of the two lower energy PL subbands increases and dominates at 400 ps delay.

\section{Discussion}

Identity of the PL emissions in c-GaN is still disputed. Only recently it was agreed that the band gap of cubic phase $\mathrm{GaN}$ is of about $3.300 \mathrm{eV}$ at $2 \mathrm{~K}$ [9]. The energies of PL transitions may differ from sample to sample, which reflects presence of strain in relatively thin GaN epilayers studied. The shift of PL position may be as large as about $10 \mathrm{meV} \mathrm{[10]} \mathrm{and} \mathrm{can} \mathrm{be} \mathrm{either} \mathrm{up} \mathrm{or} \mathrm{down} \mathrm{in} \mathrm{the} \mathrm{energy.}$ This is why identification of PL transitions in GaN epilayers is often tentative.

The present experimental results indicate a bound exciton (DBE) origin of the $3.251 \mathrm{eV}$ PL. Its intensity saturates at increased excitation intensity. This PL decreases fast with increasing temperature and shows a fast PL decay. We observed 
such properties for the DBE transition in w-GaN [11]. The $3.191 \mathrm{eV}$ PL is likely of the free-to-bound nature (hole-donor?). This identification explains most of the present experimental results.

Two low energy emissions with the maxima at $3.116 \mathrm{eV}$ and $3.034 \mathrm{eV}$ are of the same origin. They are considerably broader than two other emissions and show much longer PL decay times. We exclude here that the $3.034 \mathrm{eV}$ PL is a LO-phonon replica of the first DAP band. The two PLs show several slightly different properties excluding such their identification. For example, a different temperature dependence of the PL intensity is observed.

The present results indicate the DAP origin of the $3.116 \mathrm{eV}$ and $3.034 \mathrm{eV}$ PLs with the same shallow donor center active in the recombination transitions, being about 20-40 meV deep. Thus, two DAPs emissions are observed due to the presence of the two types of shallow acceptors with their ionization energies of about $100 \mathrm{meV}$ and $200 \mathrm{meV}$. These energies are estimated from the comparison of the spectral positions of the two PLs with the energy of relevant shallow DAP emission in w-GaN, taking into account the difference of band gaps of two phases of GaN.

Concluding, the present results confirm the recent suggestion on a smaller ionization energy of shallow acceptors in $\mathrm{c}-\mathrm{GaN}$ as compared to acceptors in $\mathrm{w}-\mathrm{GaN}$. This, as already mentioned in the introduction, is a very important property of cubic phase GaN epilayers.

This work was partly financed by joint project no. 76568 of the Committee for Scientific Research (Poland) and Ministry of Foreign Affairs (France).

\section{References}

[1] D. Ahn, S.-H. Park, Appl. Phys. Lett. 69, 3303 (1996).

[2] J. Menninger, U. Jahn, O. Brandt, H. Yang, K. Ploog, Phys. Rev. B 53, 1881 (1996).

[3] T. Lei, T.D. Moustakas, R.J. Graham, Y. He, S.J. Berkowitz, J. Appl. Phys. 17, 4933 (1992).

[4] M.J. Pansley, Z. Sitar, J.B. Posthill, R.F. David, J. Vac. Sci. Technol. B 7, 701 (1989).

[5] R.C. Powell, G.A. Tomasch, Y.W. Kim, J.A. Thorsto, J.E. Greene, Proc. MRS Symp. 162, 525 (1992).

[6] S. Miyoshi, K. Onabe, N. Ohkouchi, H. Yaguchi, R. Ito, S. Fukatsu, Y. Shiraki, J. Cryst. Growth 124, 439 (1992).

[7] A. Barski, U. Rossner, J.L. RouviCre, M. Arlery, MRS Internet Journal, Vol. 1, Article 21 (1996).

[8] R. Klann, O. Brandt, H. Yang, H.T. Grahn, K. Ploog, A. Trampert, Phys. Rev. B 52, R11615 (1995).

[9] G. Ramirez-Flores, H. Navarrow-Contreras, A. Lastras-Martinez, R.C. Powell, J.E. Greene, Phys. Rev. B 50, 8433 (1994).

[10] K. Naniwae, S. Itoh, H. Amano, K. Itoh, K. Hiramatsu, I. Akasaki, J. Cryst. Growth 99, 381 (1990).

[11] M. Godlewski, J.P. Bergman, B. Monemar, U. Rossner, A. Barski, Appl. Phys. Lett. 69, 2089 (1996). 